\title{
Phase-field modeling of the non-congruent crystallization of a ternary Ge-Sb-Te alloy for phase-change memory applications
}

\author{
R. Bayle, ${ }^{1,2}$ O. Cueto, ${ }^{1,2}$ S. Blonkowski, ${ }^{1,2}$ T. Philippe, ${ }^{3}$ H. Henry, ${ }^{3}$ and M. Plapp ${ }^{3}$ \\ 1) CEA-LETI Université Grenoble Alpes, 38000 Grenoble, France \\ 2) STMicroelectronics, 850 rue Jean Monnet, 38926 Crolles, France \\ 3) Laboratoire de Physique de la matière condensée, Ecole Polytechnique, CNRS, IP Paris, 91128 Palaiseau, \\ France
}

(Dated: 9 October 2020)

The ternary alloy of germanium, antimony, and tellurium (GST) is widely used as material for phase-change memories. In particular, the stoichiometric compound $\mathrm{Ge}_{2} \mathrm{Sb}_{2} \mathrm{Te}_{5}$ exhibits a rapid congruent crystallization. To increase the temperature at which spontaneous crystallization erases the stored information, alloys that are enriched in germanium have been investigated. Their crystallization is accompanied by segregation and eventually the nucleation of a new, germanium-rich phase. In order to model the redistribution of alloy components and the time evolution of the microstructure during device operations, we develop a multi-phasefield model for the crystallization of GST that includes segregation, and couple it with orientation fields that describe the grain structure. We demonstrate that this model is capable to capture both the emergence of a two-phase polycrystalline structure starting from an initially amorphous material, and the melting and recrystallization during the SET and RESET operations in a memory cell of the "wall" type.

Keywords: Phase-change memories; phase-field models; crystallization

\section{INTRODUCTION}

The quest for non-volatile random-access memories for information storage has led to the exploration of various solutions based on different physical effects ${ }^{1}$. Phase-Change Memory (PCM) is considered one of the most mature technologies among emerging non-volatile memories $^{2}$. In phase-change memories, each bit of information is stored in a small portion of a phase-change material, which can exist in two different solid states : crystalline and amorphous. The memory effect results from the contrast in the electrical resistivity of the two phases. To be an appropriate candidate for PCM applications, a phase-change material must on the one hand exhibit strongly different electrical resistivities in the two states, and on the other hand be able to switch rapidly between them. There are many materials that can be rapidly quenched from the melt to an amorphous state, but only very few also exhibit a pronounced difference in electrical resistivity between the amorphous and crystalline states. This contrast arises from the atomic arrangement that differs between the amorphous and crystalline states; nevertheless, the atomic rearrangement required by recrystallization can proceed on a fast timescale (ten to one hundred nanoseconds) in those materials ${ }^{3}$. The concept of using the amorphous to crystalline phase transition of chalcogenide materials to store information has been proposed since the early $1960 \mathrm{~s}^{4}$. In the $1980 \mathrm{~s}$, the research highlighted the ternary Ge-Sb-Te system with different compositions located on the pseudo-binary line GeTe-Sb $\mathrm{Te}_{3}$ and allowing crystallization in a few tens of nanoseconds ${ }^{5}$. This alloy, in particular in its stoichiometric composition $\mathrm{Ge}_{2} \mathrm{Sb}_{2} \mathrm{Te}_{5}$, originally studied for optical disk applications ${ }^{6}$, is among the most popular materials for PCM devices. With this composition, the material

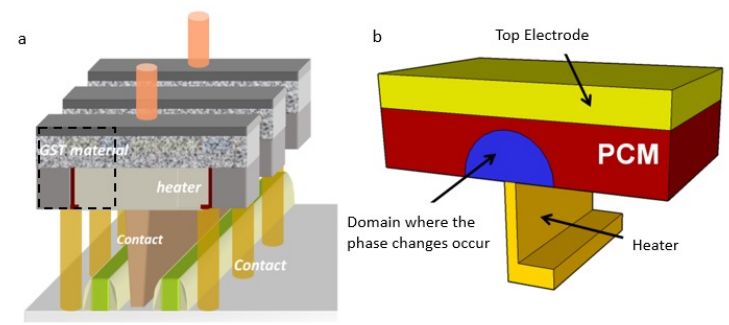

FIG. 1: Schematic view in three dimension of a technological stack including several PCM Wall cells (a) and a zoom on the Wall structure itself (b).

crystallizes rapidly without change in composition (congruent crystallization). In PCM memories, the switching between the two states is accomplished by localized heating of the material prompted by an electric current (Joule effect). Engineering of the memory cell has led to a thermally optimized structure, the so-called Wall structure ${ }^{7}$, schematized in figure 1: the cell is made of a bottom contact, a heater element for heat generation by the Joule effect, and a $\mathrm{Ge}_{x} \mathrm{Sb}_{y} \mathrm{Te}_{z}$ layer that is capped with a top contact.

The amorphous state of the PCM material is metastable, and the transition to the thermodynamically stable crystalline state always eventually occurs, with a waiting time that strongly depends on temperature. Therefore, a crystallization temperature can be defined if the required retention time is specified. The $\mathrm{Ge}_{2} \mathrm{Sb}_{2} \mathrm{Te}_{5}$ compound shows a crystallization temperature close to $150^{\circ} \mathrm{C}^{8}$. Therefore, this alloy is not suitable to guarantee data storage when functionality in an extended temperature range is requested. For example, roughly 1-2 years high temperature data retention at $150^{\circ} \mathrm{C}$ is re- 


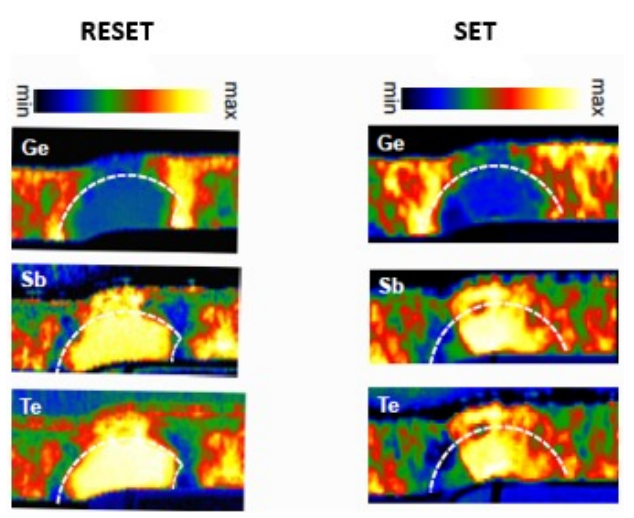

FIG. 2: Chemical mapping using STEM/EELS of a PCM Wall Structure after device operations ${ }^{13}$. The material in the active zone (area delimited by the white dashed lines) is in the amorphous and the crystalline state after the RESET and SET operations, respectively.

quested in case of automotive applications ${ }^{9}$. In order to improve the retention properties of the PCM cell based on the $\mathrm{Ge}-\mathrm{Sb}-\mathrm{Te}$ chalcogenide, the germanium-rich portion of the ternary diagram has been explored, and a germanium-rich region where the crystallization temperature is around $350^{\circ} \mathrm{C}$ has been identified ${ }^{10-12}$. The price to pay is that now the crystallization is no longer congruent: the growing GST crystal rejects germanium into the surrounding amorphous matrix. If this enrichment becomes sufficiently strong, a new phase, almost pure crystalline germanium, can appear in the system. Since GST and germanium have quite distinct electrical and thermophysical properties, the spatial distribution of the two phases has to be known in order to assess the quality and security of memory operations. Information can be obtained post mortem by electron microscopy ${ }^{11,13}$. In the example shown in Fig. 2, the redistribution of alloy components after the device operation can clearly be distinguised. However, these methods cannot give any insights concerning the dynamical processes at play. In order to better understand the fundamental mechanisms behind the evolution of composition and structure, a mathematical model that is capable to describe the dynamics of crystallization, segregation, and microstructure evolution processes in germanium-rich GST would therefore be highly useful.

In metallurgy and many other applications, phase-field models have been highly successful for the description of microstructure evolution ${ }^{14-18}$. In such models, the local state of matter is described by one or several scalar phase fields, which can be coupled to thermodynamic state variables and transport equations. Interfaces and surfaces are described in this framework by rapid but smooth variations of the phase field across diffuse interfaces with a small but finite thickness. The equations of motion for all fields can be obtained from the fundamental principles of non-equilibrium thermodynamics, and take the form of coupled partial differential equations that can be timeintegrated with standard methods. Phase-field modeling has already been applied to describe the congruent crystallization of phase-change materials ${ }^{19-21}$.

In the present contribution, we formulate a phase-field model for the crystallization of germanium-rich GST alloys that takes into account segregation and the existence of two crystalline phases. For this purpose, we combine several models that are available in the literature. The crystallization of an amorphous phase into two solids of different structures and composition is akin to an eutectic reaction; consequently, we choose a thermodynamic model which produces an eutectic phase diagram. A phase-field model for eutectic solidification ${ }^{22}$ is then adapted to the specifics of the GST alloy, using the grand-potential formalism ${ }^{23,24}$. Finally, to resolve the grain structure of both phases, two orientation fields are added to the model, following lines of earlier work on polycristalline materials ${ }^{25}$. The various parameters that appear in the model are related to physical quantities, whenever the latter are available.

The switching between amorphous and crystalline states is triggered in PCM memory cells by short current pulses, which induce through Joule heating a rapid (on the time scale of $100 \mathrm{~ns}$ ) and localized (on the scale of a memory cell of several $10 \mathrm{~nm}$ ) heating and cooling of the material. Therefore, crystallization and microstructure evolution take place in a temperature field with a non-trivial spatiotemporal structure. In order to limit power consumption while operating the memory cell, the Wall architecture was specially engineered to minimize the volume of PCM in which phase change occurs, which is called the active volume of PCM (blue area in Fig. 1b and area within white dashed lines in Fig. 2). During device operations, the PCM material in the active domain gets melted when the current is maximal, and depending on the way the current and subsequently the temperature are decreased in the active domain, the material gets the time to crystallize or not. In order to simulate a realistic proxy of a device operation, we therefore couple our phase-field model to an electrothermal solver which provides an approximation for the temperature field for a given shape of the current pulse. This temperature field is used as input data for our phase-field simulations.

The remainder of this article is structured as follows: in section II, we present the various parts of the model, discuss the choice of the parameters, and give some details on the implementation of the simulations. In section III, we present simulations of two different situations that are relevant for PCM applications: the crystallization of an as-deposited thin amorphous film, and the SET and RESET operations that are used to switch back and forth between the crystalline and amorphous states. A brief discussion of the results is given in Section IV, followed by conclusions and perspectives. 


\section{MODEL}

\section{A. Approximations}

The crystallization of a ternary mixture under nonequilibrium conditions is a complex process. In order to obtain a tractable problem, we make a certain number of approximations:

1. In a ternary mixture, there are two independent degrees of freedom for the composition. However, the composition maps obtained from TEM analyses shown in figure 2 indicate that there is a strong correlation between the concentrations of $\mathrm{Te}$ and $\mathrm{Sb}$. Therefore, we make a pseudobinary approximation: we consider only alloys that are located on a linear segment in the ternary phase diagram that extends between stoichiometric $\mathrm{Ge}_{2} \mathrm{Te}_{2} \mathrm{Sb}_{5}$ and pure germanium. We define a scaled concentration variable $c$ that describes the position on this segment, with $c=0$ corresponding to the stoichiometric compound and $c=1$ to pure germanium.

2. In the ternary alloy GST, a large number of stoichiometric compounds have been identified, and the phase diagram is complex ${ }^{26}$. X-ray diffraction spectra measured on thin layers of GST alloy enriched with germanium after annealing at $400^{\circ} \mathrm{C}$ show the presence of two cubic phases identified as the 225 and Ge phases ${ }^{27}$. Therefore, among all the stoichiometric compounds we will only take into account the 225 compound and call it GST phase. The other phases present in the system are crystalline germanium and the amorphous phase. Since the relevant temperatures for device crystallization operation are located between the glass temperature of $80^{\circ} \mathrm{C}^{28}$ and the equilibrium temperature for crystallization, the amorphous phase will be assimilated to a supercooled liquid with very slow kinetics.

3. We will assume that the molar volume of the three involved phases (GST, germanium and amorphous) is the same. This is an approximation commonly used in the field of phase-field modeling. In particular, this implies that we do not take into account mechanical effects on the phase-change mechanism.

4. In the calculations of the temperature field, we do not take into account the release of the latent heat of crystallization. This is reasonable as a first approximation, given that the adiabatic temperature (the ratio of latent heat and specific heat) for GST is approximately $480 \mathrm{~K}$, whereas the temperature changes generated by the Joule heating are of the order of $1000 \mathrm{~K}$. This issue will be further discussed below.

These approximations, together with a large degree of uncertitude on several materials parameters (see below) imply that we cannot expect the model to be quantitatively accurate. Nevertheless, as we will see, the model is capable to account for numerous observations made in the experiments.

\section{B. Thermodynamic model}

The phase-field method needs as an input the freeenergy density of each phase as a function of composition and temperature. Since free-energy data are hard to obtain directly, we adopt a similar approach as the CALPHAD method, which uses physically motivated models for the free energy and adjusts the parameters of these models to available data, such as phase diagrams and calorimetric data ${ }^{29}$. Our starting point is the pseudobinary phase diagram of Ref. ${ }^{26}$ along a line reaching from $\mathrm{Sb}_{2} \mathrm{Te}_{3}$ to pure germanium, which is close to the segment that we consider. On the two sides (stoichiometric compound and pure germanium), a solid-liquid coexistence occurs, with the behavior that is characteristic for dilute solutions. In particular, the melting temperature of the GST phase decreases upon addition of germanium, and the melting temperature of the germanium phase decreases upon addition of Te and Sb. Moreover, in thermal annealing of amorphous films, it is observed that the germanium phase nucleates first and grows, thereby decreasing the germanium content of the amorphous matrix, until the GST phase also nucleates and grows ${ }^{30,31}$. The simplest phase diagram that is compatible with these facts is of the eutectic type. Therefore, we choose free-energy functions that generate an eutectic phase diagram. This is done by considering two regular solution models with simple lens-shape phase diagrams, one between GST-225 and the virtual compound $\mathrm{X}$, and the other between germanium and the virtual compound $Y$. The properties of the virtual compounds $\mathrm{X}$ and $\mathrm{Y}$ are then adjusted such as to obtain the correct liquidus and solidus slopes in the dilute limits, and a reasonable value for the eutectic temperature and composition.

The free-energy functions for the GST, germanium and liquid (amorphous) phases are, respectively,

$$
f_{G S T}(c, T)=\frac{1}{V_{m}}\left((1-c) \frac{L_{G S T}}{T_{m}^{G S T}}\left(T-T_{m}^{G S T}\right)+c \frac{L_{X}}{T_{m}^{X}}\left(T-T_{m}^{X}\right)+R T(c \ln (c)+(1-c) \ln (1-c))+\Omega_{G S T} c(1-c)\right)
$$




$$
\begin{gathered}
f_{G e}(c, T)=\frac{1}{V_{m}}\left((1-c) \frac{L_{Y}}{T_{m}^{Y}}\left(T-T_{m}^{Y}\right)+c \frac{L_{G e}}{T_{m}^{G e}}\left(T-T_{m}^{G e}\right)+R T(c \ln (c)+(1-c) \ln (1-c))+\Omega_{G e} c(1-c)\right) \\
f_{l i q}(c, T)=\frac{1}{V_{m}}\left(R T(c \ln (c)+(1-c) \ln (1-c))+\Omega_{l i q} c(1-c)\right)
\end{gathered}
$$

where $T_{m}^{i}$ is the melting temperature of pure compound (or element) $i, L_{i}$ its molar latent heat of melting, and $\Omega_{i}$ its molar enthalpy; furthermore, $c$ is the scaled germanium concentration, $R$ is the gas constant, $T$ the temperature, and $V_{m}$ the molar volume.

The enthalpy coefficient of the liquid, $\Omega_{l i q}$ is chosen such as to make the liquid free energy convex; the enthaly coefficients of the Ge and GST phases can then be related to the partition coefficients (the ratios of the liquidus and solidus slopes in the phase diagram at the respective melting points) $k_{G e}$ and $k_{G S T}$ through

$\Omega_{G S T}=\Omega_{l i q}-R T_{m}^{G S T} \ln \left(k_{G S T}\left(T_{m}^{G S T}\right)\right)-\frac{L_{X}}{T_{m}^{X}}\left(T_{m}^{G S T}-T_{m}^{X}\right)$

$$
\Omega_{G e}=\Omega_{l i q}-R T_{m}^{G e} \ln \left(k_{G e}\left(T_{m}^{G e}\right)\right)+\frac{L_{Y}}{T_{m}^{Y}}\left(T_{m}^{G e}-T_{m}^{Y}\right),
$$

where we have chosen the latent heats of the virtual compounds to satisfy $L_{X} / T_{m}^{X}=L_{G S T} / T_{m}^{G S T}$ and $L_{Y} / T_{m}^{Y}=$ $L_{G e} / T_{m}^{G e}$. The values of the constants used are listed in table I. The phase diagram is obtained from these functions through the standard common tangent construction and is shown in figure 3 .

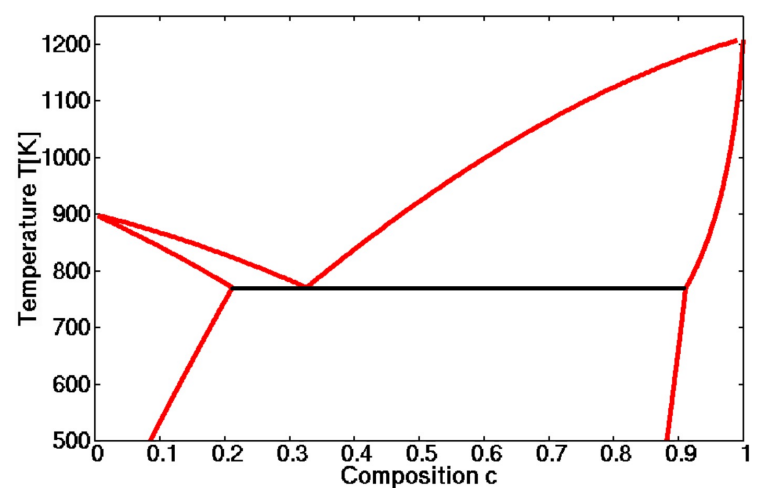

FIG. 3: Phase diagram obtained from the free energy functions with the parameters listed in table I.

\section{Multi-phase-field model for crystallization}

We use a multi-phase field model that was originally developed for the description of eutectic solidification ${ }^{22}$, together with a grand-canonical reformulation of phasefield models for alloy solidification ${ }^{23,24}$. The model works with three phase fields $p_{i}$, each of which represents the local volume fraction of phase $i$. Therefore, they satisfy the constraint $\sum_{i} p_{i}=1$. We associate $p_{1}$ with the GST phase, $p_{2}$ with the germanium phase, and $p_{3}$ with the amorphous phase. The model equations can be obtained from the grand-potential functional

$$
\Omega=\int_{V} \omega(\mathbf{p}, \vec{\nabla} \mathbf{p}, \mu, T) d V
$$

where $\omega$ is the grand-potential density, which depends on all the phase fields, their gradients as well as on temperature and the chemical (diffusion) potential; we denote by the bold face $\mathbf{p}$ the set of the three phase fields $\left\{p_{1}, p_{2}, p_{3}\right\}$. The grand-potential density reads

$$
\begin{aligned}
\omega(\mathbf{p}, \vec{\nabla} \mathbf{p}, \mu, T)= & K \omega_{\text {grad }}(\vec{\nabla} \mathbf{p}) \\
& +H \omega_{\mathrm{TW}}(\mathbf{p})+\omega_{\mathrm{th}}(\mathbf{p}, \mu, T) .
\end{aligned}
$$

The second term, $\omega_{\mathrm{TW}}$, is a triple-well potential given by

$$
\omega_{\mathrm{TW}}(\mathbf{p})=\sum_{i} p_{i}^{2}\left(1-p_{i}\right)^{2}
$$

that has three wells of equal depth, which correspond to the three possible phases. In each well, the value of one phase field is equal to unity, whereas the others are equal to zero. The first term of Eq. (7) contains gradient terms,

$$
\omega_{\text {grad }}(\vec{\nabla} \mathbf{p})=\frac{1}{2} \sum_{i}\left(\vec{\nabla} p_{i}\right)^{2}
$$

which penalize spatial variations of the phase fields and ensure that the variations of the phase fields between any two phases are continuous.

The third term in Eq. (7) represents the thermodynamic driving force for phase transformations, which modifies the depth of each well according to the local thermodynamic conditions. In the early phase-field models for alloy solidification, this term was directly written as a function of the composition $c$. However, in a mixture, two phases in equilibrium do generally not have the same composition; this renders the determination of the driving force in terms of the composition complicated ${ }^{33}$. It is easier to express it in terms of the grand potential densities of the individual phases,

$$
\omega_{i}(\mu, T)=f_{i}(c, T)-\mu c,
$$

where

$$
\mu=\frac{\partial f_{i}}{\partial c}
$$




\begin{tabular}{|c|c|c|}
\hline$V_{m}$ & molar volume & $1.6 \times 10^{-5} \mathrm{~m}^{3} /$ mole \\
\hline$T_{m}^{G e}$ & melting temperature of Ge & $1211 \mathrm{~K}$ \\
\hline$T_{m}^{G S T}$ & melting temperature of GST & $900 \mathrm{~K}$ \\
\hline$L_{G e}$ & heat of fusion of Ge & $3.7 \times 10^{4} \mathrm{~J} /$ mole \\
\hline$L_{G S T}$ & heat of fusion of GST & $1.2 \times 10^{4} \mathrm{~J} / \mathrm{mole}$ \\
\hline$\Omega_{l i q}$ & enthalpy coefficient of the liquid & $1.5 \times 10^{4} \mathrm{~J} / \mathrm{mole}$ \\
\hline$T_{m}^{X}$ & melting temperature of virtual compound X & $150 \mathrm{~K}$ \\
\hline$T_{m}^{Y}$ & melting temperature of virtual compound Y & $440 \mathrm{~K}$ \\
\hline$k_{G S T}$ & partition coefficient of GST & 0.5 \\
\hline$k_{G e}$ & partition coefficient of Ge & $1.9 \times 10^{-2}$ \\
\hline
\end{tabular}

TABLE I: Values of the parameters in the free energy model ${ }^{26,32}$.

is the diffusion potential, which is the thermodynamic conjugate of the composition $c$. The grand potential densities $\omega_{i}$ are thus the Legendre transforms of the free energy functions $f_{i}$. At equilibrium, the diffusion potentials and the grand potentials of two coexisting phases are equal; outside of equilibrium, the driving force for interface motion is given by the difference between the grand potential densities of the two phases. For freeenergy functions as given by Eq. (1), which are transcendental in $c$, the Legendre transform cannot be calculated analytically, but it can easily be tabulated numerically.
This procedure is without difficulty as long as the relation between $\mu$ and $c$ is monotonic, which is the case for all three phases in our system. Once the three grandpotential densities are determined, the driving-force term can be expressed as

$$
\omega_{\mathrm{th}}(\mathbf{p}, \mu, T)=\sum_{i} g_{i}(\mathbf{p}) \omega_{i}(\mu, T)
$$

with interpolation functions $g_{i}(\mathbf{p})$ defined in Ref. ${ }^{22}$,

$$
g_{i}\left(p_{i}, p_{j}, p_{k}\right)=\frac{p_{i}^{2}}{4}\left\{15\left(1-p_{i}\right)\left[1+p_{i}-\left(p_{j}-p_{k}\right)^{2}\right]+p_{i}\left(9 p_{i}^{2}-5\right)\right\}
$$

where the values of the indices $i, j$, and $k$ are all different. These functions satisfy $g_{i}\left(p_{i}=1\right)=1$, and $g_{i}\left(p_{i}=0\right)=$ 0.

The evolution equations for the phase fields are simple relaxation equations that generalize the Allen-Cahn equation $^{34}$ and are obtained from the variational principle, taking into account the sum constraint on the phase fields through a Lagrange multiplier ${ }^{22}$,

$$
\begin{aligned}
\frac{\partial p_{i}}{\partial t} & =-\left.\Gamma \frac{\delta \Omega}{\delta p_{i}}\right|_{\sum_{i} p_{i}=1} \\
& =-\Gamma\left(\frac{2}{3} \frac{\delta \Omega}{\delta p_{i}}-\frac{1}{3} \frac{\delta \Omega}{\delta p_{j}}-\frac{1}{3} \frac{\delta \Omega}{\delta p_{k}}\right),
\end{aligned}
$$

where $\Gamma$ is a relaxation rate constant. Given the respective dimensions of the constants $K, H$, and $\Gamma$, the interface width $W$ and relaxation time $\tau$ can be defined by

$$
\begin{aligned}
W & =\sqrt{\frac{K}{H}}, \\
\tau & =\frac{1}{\Gamma H} .
\end{aligned}
$$

After evaluation of the functional derivatives, the evolution equation reads

$$
\begin{aligned}
\tau \frac{\partial p_{i}}{\partial t}= & W^{2} \nabla^{2} p_{i} \\
& -\frac{2}{3}\left(\sum_{j}\left(3 \delta_{i j}-1\right) p_{j}\left(1-p_{j}\right)\left(1-2 p_{j}\right)\right) \\
& -\left.\frac{1}{H} \sum_{j} \omega_{j}(\mu) \frac{\partial g_{j}}{\partial p_{i}}\right|_{\sum_{i} p_{i}=1} .
\end{aligned}
$$

The concentration obeys a conservation law,

$$
\frac{\partial c}{\partial t}=-\vec{\nabla} \cdot \vec{J}
$$

with a diffusive mass current driven by the diffusion potential gradient,

$$
\vec{J}=-M(\mu, T) \vec{\nabla} \mu,
$$

with $M(\mu, T)$ the interdiffusion mobility of germanium. Since, in the grand-canonical framework, the composition is a quantity that derives variationally from the grand potential,

$$
c(\mu, T, \mathbf{p})=-\frac{\delta \Omega}{\delta \mu}
$$


Eq. (18) can be transformed into an evolution equation for $\mu$ using the chain rule ${ }^{23,24}$. However, since the relation between $\mu$ and $c$ is nonlinear, the numerical errors linked to time discretization lead to a slight violation of mass conservation. In order to avoid these numerical errors, we use a mixed formulation, in which both the fields $c$ and $\mu$ are stored. The concentration is then evolved using Eq. (18), and the value of $\mu$ compatible with the new values of $c$ and $\mathbf{p}$ is found by a simple numerical scheme that converges in a few iterations.

\section{Coupling to orientation fields}

In the experiments, it is observed that the crystallization of the amorphous phase generally leads to polycrystalline materials. Therefore, the model should be able to describe crystal grains and grain boundaries. Two strategies have been developed in phase-field models to achieve this. The first is to associate a separate phase field to each grain of the crystalline phases. The second strategy is to use orientation fields, which indicate the local orientation of the crystalline unit cell with respect to a fixed reference frame. For a single crystalline phase, in two dimensions a single scalar orientation field is sufficient ${ }^{25,35}$, whereas in three dimensions several fields are necessary ${ }^{36,37}$. Grain boundaries correspond to localized variations of the orientation field.

Whereas the orientation-field formalism is numerically simpler, it has, to our knowledge, not previously been used in conjunction with multi-phase-field models. We present here a first attempt to formulate such a model. Whereas we have not succeeded to put together a model that is fully variational, the results presented below demonstrate that this model constitutes a reasonable phenomenological description.

Our starting point is the two-dimensional single-phase orientation-field model of Ref. ${ }^{25}$, which uses a singular coupling between orientation and phase fields. Indeed, rotational invariance imposes that the free energy cannot depend on the orientation field, but only on its gradients. The singular dependence of the coupling ensures that the energetic cost of orientation variations diverges in the solid, whereas it vanishes in the liquid. As a result, the orientation field is almost constant within the crystalline grains, whereas it rapidly varies across grain boundaries. In the center of the grain boundaries, a small fraction of liquid always remains present.

We generalize this model by introducing two separate orientation fields $\theta_{1}$ and $\theta_{2}$ for the GST and germanium crystalline phases. The grand potential is supplemented by an orientational part,

$$
\begin{aligned}
\omega_{\text {ori }}(\mathbf{p}, \vec{\nabla} \theta)= & C_{\theta}\left[q\left(p_{1}\right)\left(\vec{\nabla} \theta_{1}\right)^{2}\right. \\
& \left.+q\left(p_{2}\right)\left(\vec{\nabla} \theta_{2}\right)^{2}\right],
\end{aligned}
$$

where the function $q(p)$ is given by $^{25,38}$

$$
q\left(p_{i}\right)=\frac{7 p_{i}^{3}-6 p_{i}^{4}}{\left(1-p_{i}\right)^{3}}
$$

and $C_{\theta}$ is a constant that determines the grain boundary energies.

If this expression is added to the grand-potential density, Eq. (7) and injected into the variational formalism, Eq. (14), undesirable properties result. Namely, the dynamics of the GST phase is influenced by the orientation field of the germanium phase, and vice versa. This results from the coupling due to the Lagrange multiplier associated with the sum constraint on the phase fields. Rather than following this route, we have preferred to couple each orientation field to the equation of motion of the corresponding phase field exactly as in the original model of Ref. ${ }^{25}$. This corresponds to taking, for the orientational term, the variational derivative without the sum constraint. The resulting equations of motion read

$$
\begin{aligned}
\tau \frac{\partial p_{i}}{\partial t}= & W^{2} \nabla^{2} p_{i} \\
& -\frac{2}{3}\left(\sum_{j}\left(3 \delta_{i j}-1\right) p_{j}\left(1-p_{j}\right)\left(1-2 p_{j}\right)\right) \\
& -\left.\frac{1}{H} \sum_{j} \omega_{j}(\mu) \frac{\partial g_{j}}{\partial p_{i}}\right|_{\sum_{i} p_{i}=1} \\
& -\frac{C_{\theta}}{H} q^{\prime}\left(p_{i}\right)\left(\nabla \theta_{i}\right)^{2}
\end{aligned}
$$

for the phase fields $i=1,2$. The field $p_{3}$ is then determined by the sum constraint.

Since the two orientation fields are not explicitly coupled, their evolution equation can be obtained by the standard variational method,

$$
\begin{aligned}
\frac{\partial \theta_{i}}{\partial t} & =-\Gamma_{\theta_{i}} \frac{\delta \Omega}{\delta \theta_{i}} \\
& =\frac{\Gamma_{\theta_{i}} C_{\theta}}{q\left(p_{i}\right)} \vec{\nabla} \cdot\left[q\left(p_{i}\right) \vec{\nabla} \theta_{i}\right],
\end{aligned}
$$

where $i=1,2$, and $\Gamma_{\theta_{i}}$ are relaxation rate constants for the orientation fields. More detailed comments and discussions about this equation of motion and its use for the description of polycrystalline single-phase materials can be found in Refs. ${ }^{25,38,39}$.

\section{E. Choice of the model parameters}

The model contains numerous parameters that need to be determined. The first are the constants $K$ and $H$ in the grand-potential density, Eq. (7). Those can be related to the thickness of the diffuse interfaces $W$, already introduced in Eq. (15), and to the interface free energy $\sigma$ by $\sigma=\sqrt{2 K H} / 3^{22}$. We choose the values $W=0.5 \mathrm{~nm}$, which is a physically realistic value for microscopically rough interfaces in metallic alloys ${ }^{40}$, and $\sigma=0.4 \mathrm{~J} / \mathrm{m}^{2}$, 
which is the value for the solid-liquid surface free energy of germanium at $650 \mathrm{~K}$ reported in Ref. ${ }^{41}$. From the values of $W$ and $\sigma$ the constants $K$ and $H$ can be calculated. It should be noted that in the present formulation, all interfaces have the same energy, which is certainly not true in the real system. However, in the absence of more detailed information, we adopt this approximation since it optimizes the model performance. This choice could easily be modified in future work following the lines of Ref. $^{22}$.

The next parameter to be discussed is the phase-field relaxation rate $\Gamma$, or equivalently the phase-field relaxation time $\tau$. It is related to the interface kinetics. No information about the interface kinetics in germaniumrich GST is available to our knowledge; however, for the stoichiometric 225 compound, the crystallization rate has been measured for a large range of temperatures by ultrafast calorimetry ${ }^{32}$. We use these data in the following way. In the absence of coupling to the composition, the driving force for phase transformation depends only on temperature. For the standard Allen-Cahn equation, the relation between the driving force (which is the free energy difference $\Delta f$ in the absence of composition coupling) and interface velocity is known ${ }^{42}$ and can be inverted to deduce the relaxation time from the interface velocity:

$$
\tau=\frac{\Delta f}{v \sigma} W^{2} .
$$

The driving force can be evaluated for GST 225 by the model of Thompson and Spaepen ${ }^{28,32}$,

$$
\Delta f=\frac{\Delta G_{m}}{V_{m}}=\frac{\Delta H_{m}\left(T-T_{m}\right)}{V_{m} T_{m}} \frac{2 T}{T+T_{m}},
$$

where $\Delta G_{m}, V_{m}, \Delta H_{m}$ et $T_{m}$ are the molar Gibbs free energy difference, the molar volume, the latent heat of fusion and the melting temperature, respectively. The numerical values used for these quantities are listed in table II at a temperature of $673 \mathrm{~K}$. This calculation is repeated for several temperatures, with the values of the interface velocity tabulated from Ref. ${ }^{32}$ and listed in table III. For other temperatures, the data points were linearly interpolated.

\begin{tabular}{|c|c|}
\hline$W$ & $5 \times 10^{-10} \mathrm{~m}$ \\
\hline$\Delta H_{m}$ & $12 \times 10^{3} \mathrm{~J} / \mathrm{mole}$ \\
\hline$T_{m}$ & $900 \mathrm{~K}$ \\
\hline$\sigma$ & $0.4 \mathrm{~J} / \mathrm{m}^{2}$ \\
\hline$v$ & $2.8 \mathrm{~m} / \mathrm{s}$ \\
\hline$\Delta f$ & $1.6^{8} \mathrm{~J} / \mathrm{m}^{3}$ \\
\hline
\end{tabular}

TABLE II: Values needed for the evaluation of $\tau$ through Eq. (25) at $673 \mathrm{~K}$.

Concerning the equation for the concentration, the mobility needs to be determined. Data for the diffusion coefficients of germanium in the crystalline and amorphous

\begin{tabular}{|c|c|}
\hline Température $T(\mathrm{~K})$ & Velocity $v(\mathrm{~m} / \mathrm{s})$ \\
\hline 400 & $10^{-7}$ \\
\hline 450 & $10^{-2}$ \\
\hline 500 & 0.45 \\
\hline 550 & 1.1 \\
\hline 600 & 2.5 \\
\hline 650 & 2.75 \\
\hline 700 & 2.6 \\
\hline 750 & 2.5 \\
\hline 800 & 2 \\
\hline 850 & 0.9 \\
\hline 900 & 0 \\
\hline
\end{tabular}

TABLE III: Interface velocity of GST 225 for different temperatures. Data extracted from Orava et al. ${ }^{32}$.

phases has been published in Ref. ${ }^{43}$. They can be well fitted by Arrhenius laws,

$$
\begin{gathered}
D_{1}(T)=D_{2}(T)=10^{\left[-4.62-0.22 \mathrm{eV} /\left(k_{B} T\right)\right]} \mathrm{cm}^{2} / \mathrm{s}, \\
D_{3}(T)=10^{\left[-3.73-0.094 \mathrm{eV} /\left(k_{B} T\right)\right]} \mathrm{cm}^{2} / \mathrm{s}
\end{gathered}
$$

where $k_{B}$ is Boltzmann's constant. Note that the order of magnitude for the diffusivities around $700 \mathrm{~K}$ is $10^{-12} \mathrm{~m}^{2} / \mathrm{s}$ for the crystalline phases and $10^{-9} \mathrm{~m}^{2} / \mathrm{s}$ for the liquid.

The mobility and the diffusion coefficients are related by the well-known relation ${ }^{44} D=M \partial \mu / \partial c$. In the grand-potential framework, we define the susceptibility function $\chi=\partial c / \partial \mu$, and find that $M=D \chi$. The susceptibility function for each phase can be found from the free-energy functions. The diffusion coefficient and the susceptibility are then interpolated according to

$$
\begin{aligned}
D(\mathbf{p}) & =g\left(p_{1}\right) D_{1}+g\left(p_{2}\right) D_{2}+g\left(p_{3}\right) D_{3}, \\
\chi(\mathbf{p}, \mu) & =g\left(p_{1}\right) \chi_{1}(\mu)+g\left(p_{2}\right) \chi_{2}(\mu)+g\left(p_{3}\right) \chi_{3}(\mu) .
\end{aligned}
$$

Finally, we also need to fix the parameters related to the orientation fields. The coupling constant in the orientational energy, Eq. (21), $C_{\theta}$, determines the grain boundary energies. The latter depends on the misorientation between the two neighboring grains. We measure the angles on a scale between 0 and 1 and fix $C_{\theta} / H=1.3 \times 10^{-21} \mathrm{~m}^{2}$, which yields a grain-boundary energy for high-angle grain boundaries (with the maximal misorientation of 0.5) comparable to the interfacial energies. This entails that finite contact angles are observed at the intersections between grain boundaries and interfaces. The evolution equations for the orientation fields also contain relaxation rates. Those are set to high values, so that the orientation fields are able to relax over the entire crystal-amorphous interface before crystallization is completed and no "disorder trapping" occurs ${ }^{45,46}$. We define relaxation times for the orientation fields by relations analogous to Eq. (16) and set $\tau_{\theta_{1}}=10^{-7} \tau(T)$ and $\tau_{\theta_{2}}=5 \times 10^{-7} \tau(T)$. 


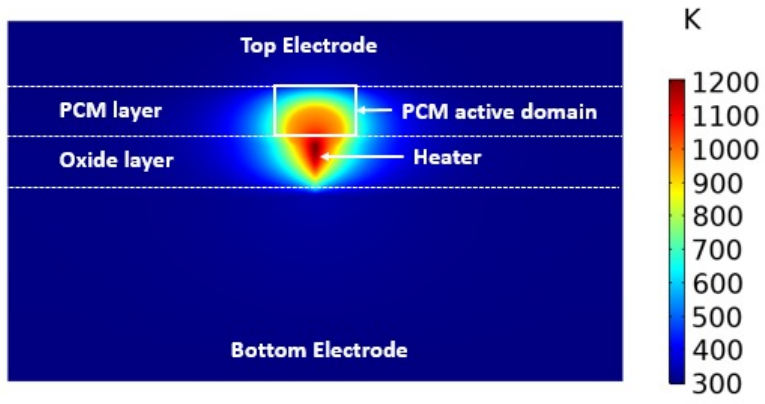

FIG. 4: Joule heating simulation of the Wall Structure. The domain in which the phase-field simulations are carried out is the rectangle delimited by the white frame.

\section{F. Coupling with an electrothermal solver}

An electrothermal simulation is realized for a PCM Wall structure including bottom and top electrodes, heater and surrounding oxides. Ambient temperature is imposed on the bottom electrode which is a thick metallic layer located under the heater and on the top electrode. The top electrode is grounded and a current is imposed on the bottom electrode to supply a given current in the structure. Insulation boundary conditions are applied on the lateral sides of the structure. Joule heating is simulated in the whole structure and a restriction of the temperature field to the active domain is used in the multi-phase-field simulation (see Fig. 4). An ohmic model approach is used to simulate the electrical behavior of the PCM cell. The electro-thermal solver relies on the coupled system of partial differential equations formed by the charges conservation equation and the heat transfer equation.

$$
\begin{gathered}
\nabla \cdot(-\sigma \nabla V)=0 \\
\rho C_{p} \frac{\partial T}{\partial t}+\nabla \cdot\left(-k_{t h} \nabla T\right)=\sigma(\nabla V)^{2}
\end{gathered}
$$

where $\sigma, \rho, C_{p}$ and $k_{t h}$ stand for the materials electrical conductivity, density, heat capacity and thermal conductivity. Electrical and thermal conductivity of the Ge rich GST are modeled as function of temperature:

$$
\begin{array}{r}
\sigma_{\mathrm{pcm}}=\frac{\sigma_{0}}{2}\left(\tanh \left(B_{e} T+C_{e}\right)+D_{e}\right) \\
k_{t h, p c m}=\frac{k_{t h}^{0}}{2}\left(\tanh \left(B_{t h} T+C_{t h}\right)+D_{\mathrm{th}}\right)
\end{array}
$$

A set of parameters for electrical and thermal conductivity for Ge-rich GST was fixed (see Table IV) in order to obtain current-voltage curves close to experimental results. The density and heat capacity used for Ge-rich
TABLE IV: Parameters for Ge-rich GST electrical and thermal conductivity

\begin{tabular}{|c|c|c|c|}
\hline$\sigma_{0}$ & $B_{e}$ & $C_{e}$ & $D_{e}$ \\
\hline $2.8 \cdot 10^{4} S / m$ & $0.0022 K^{-1}$ & -1.8 & 1 \\
\hline$k_{t h}^{0}$ & $B_{t h}$ & $C_{t h}$ & $D_{t h}$ \\
\hline $2.566 \mathrm{~W} / \mathrm{K} / \mathrm{m}$ & $0.0051 K^{-1}$ & -48.359 & 1.418 \\
\hline
\end{tabular}

GST are those of GST, taken from Ref. ${ }^{47}: \rho=6350$ $\mathrm{kg} / \mathrm{m}^{3}$ and $C_{p}=24.9 \mathrm{~J} /($ mole $\mathrm{K})$. They are taken at 300 $\mathrm{K}$ and considered as independent of temperature.

This calculation of the thermal field does not take into account the release of the latent heat of crystallization. Using the value of the latent heat from Table II, the adiabatic temperature defined by $T_{Q}=L / C_{p}$ is estimated as $482 \mathrm{~K}$. This is the temperature range associated with the phase transition. It is significantly smaller than the temperature changes of the order of $1000 \mathrm{~K}$ obtained by the Joule heating in the simulation (see Fig. 4). In a full two-way coupling between phase-field and electrothermal model, it would be straightforward to include in the thermal model a source term for the release of latent heat, which is proportional to the change with time of the phase fields. However, due to the very different diffusivities of heat and chemical components, this results in a problem which is numerically exceedingly stiff. Therefore, we have chosen not to include this effect for the time being.

This system of equations is discretized using the FiniteElement method with the Partial Derivative Equation interface of COMSOL multiphysics®. In PCM materials, electrical and thermal conductivities depend on the temperature. This leads to a strongly coupled and nonlinear set of equations which is solved self-consistently using a nonlinear Newton solver.

\section{G. Implementation and simulation setup}

We perform our phase-field calculations in two dimensions, and focus on a domain that corresponds to the PCM active domain visible in Fig. 4. This corresponds to a cut through the thin film of phase-change material perpendicular to the heater electrode and larger than the blue hemicircle in the sketch of Fig. 1. The domain size is $150 \mathrm{~nm} \times 100 \mathrm{~nm}$. The evolution equations for the phase-fields, the concentration field and the orientation fields are discretized using the finite-differences method on a regular Cartesian grid of grid spacing $\Delta x=0.25 \mathrm{~nm}$ (in total, $601 \times 401$ grid points). All the equations are time-stepped using a simple explicit Euler scheme with time step $\Delta t=7.8 \times 10^{-13} \mathrm{~s}$. In the lateral directions, periodic boundary conditions are applied; in the vertical direction, zero-gradient Neumann boundary conditions are used. Three different situations are considered: isothermal heat treatment, for which the temperature is set to 
a constant, and the SET and RESET operations. Heat maps calculated by the electrothermal solver are stored for each grid point at regular time intervals of $50 \mathrm{~ns}$ for the SET pulse and 5 ns for the RESET pulse. Time sampling is different for the pulses due to their different duration (5.4 $\mu$ s for the SET pulse and $110 \mathrm{~ns}$ for the RESET pulse). These temperature fields are then used to evaluate all free energies and mobilities in the phase-field calculations, with linear interpolation of the temperature for times in between two stored maps.

\section{SIMULATION RESULTS}

\section{A. Isothermal crystallization of an amorphous thin film}

The first situation that has been simulated is the crystallization of an initially amorphous Ge-rich GST layer. The model presented in the previous section is deterministic and does not include nucleation events. Therefore, in order to initialize crystallization, nucleation has to be explicitly modeled. A nucleation scenario deduced from experimental results ${ }^{30,31}$ has been used. In this scenario, the Ge phase appears first and starts to grow. The growth of the germanium grains decreases the Ge concentration in the surrounding amorphous phase, which makes the nucleation of the GST phase possible. The (homogeneous) nucleation rates of GST can be evaluated from classical nucleation theory. This yields rates that depend very strongly on the composition of the amorphous matrix and that increase with decreasing Ge concentration. The probability for nucleation within one time step becomes unity for $c<0.35$; therefore, we initialize nucleation of the GST phase as soon as the concentration falls below this threshold, under condition that there is no crystalline phase in the neighborhood of the freshly introduced nucleus.

Snapshot pictures of the structural order are represented in figure 5. Initially (a), a set of randomly placed nuclei with random orientation of Ge phase are introduced simultaneously. After $590 \mathrm{~ns}$ (b), the Ge grains have grown and impoverished in Ge the amorphous phase. After $830 \mathrm{~ns}$ (c), some GST nuclei have appeared and grown. After those nucleation events, both crystalline phases grow at the expense of the amorphous phase; this is still ongoing at $1.6 \mu \mathrm{s}(\mathrm{d})$. At $1.9 \mu \mathrm{s}$, the amorphous phase has disappeared. But this does not mean that the structural evolution has stopped : small grains tend to dissolve into bigger ones due to Ostwald ripening, and grain boundaries between Ge grains tend to be eliminated (f). In this stage, since the amorphous phase, which has the fastest diffusion kinetics, has disappeared, it was possible to increase the timestep by a factor of 1000. Nevertheless, the maximal simulated time remains much shorter than typical annealing times in experiments.

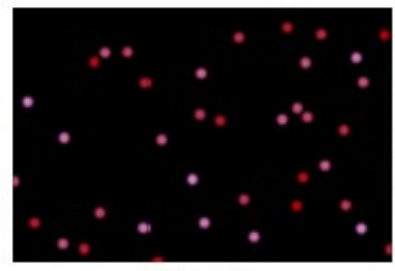

(a) $\mathrm{t}=0 \mathrm{~s}$

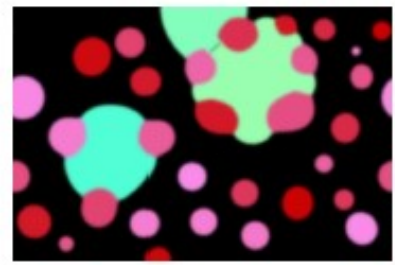

(c) $\mathrm{t}=830 \mathrm{~ns}$

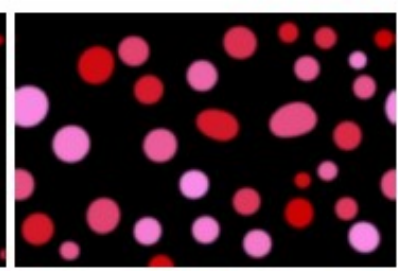

(b) $\mathrm{t}=590 \mathrm{~ns}$

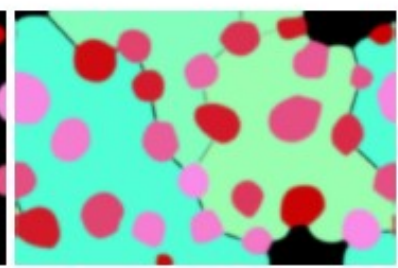

(d) $\mathrm{t}=1.6 \mu \mathrm{s}$

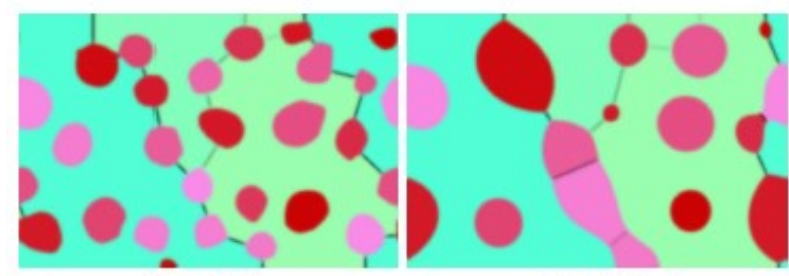

(e) $\mathrm{t}=1.9 \mu \mathrm{s}$

(f) $\mathrm{t}=5.7 \mathrm{~ms}$

FIG. 5: Snapshot pictures of the crystalline microstructure at times $0 \mathrm{~s} \mathrm{(a),} 590 \mathrm{~ns}$ (b), $830 \mathrm{~ns}$ (c), $1.6 \mu \mathrm{s}(\mathrm{d}), 1.9 \mu \mathrm{s}(\mathrm{e})$, and $5.7 \mathrm{~ms}(\mathrm{f})$ in a $2 \mathrm{D}$ simulation of an initially amorphous layer of Ge-rich GST.

Amorphous areas are represented in black. Ge and GST crystalline phases are respectively represented in pink/red and green/blue. The different shades of pink/red and green/blue stand for the different orientations of nuclei.

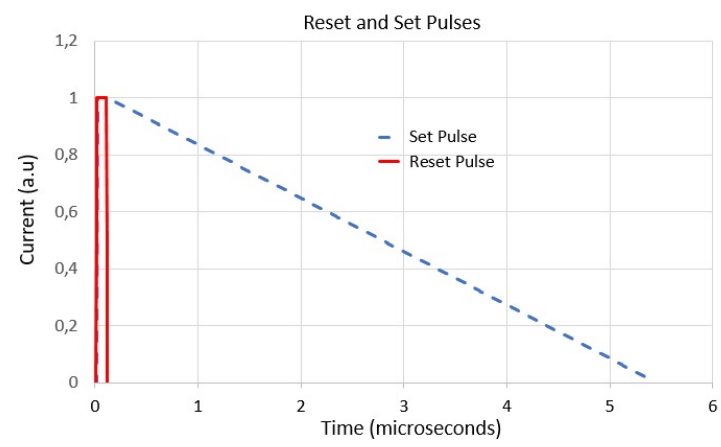

FIG. 6: Electric current (arbitrary units) as a function of time for the RESET and SET operations, respectively associated with the red and blue curves.

\section{B. RESET operation}

The final state of the isothermal simulation is a polycrystalline two-phase material that can be used as an initial condition to study the effect of PCM operations 
on the microstructure. We use the coupled approach described previously. First, we simulate the RESET operation, which corresponds to a short intense current pulse followed by rapid cooling that brings the material in its amorphous state.

The time profile of the current pulse used for the RESET operation is plotted in figure 6 as the red line. The thermal fields obtained with the electrothermal model are represented for various times in figure 7 .

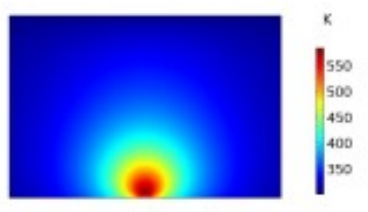

(a) $\mathrm{t}=5 \mathrm{~ns}$

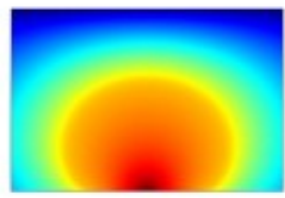

(c) $\mathrm{t}=100 \mathrm{~ns}$

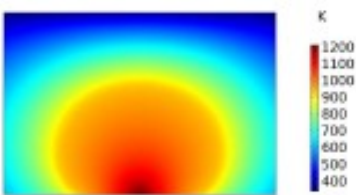

(b) $\mathrm{t}=30 \mathrm{~ns}$

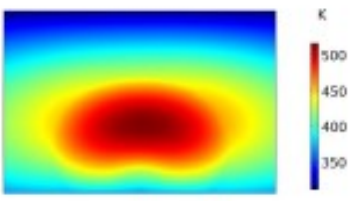

(d) $\mathrm{t}=110 \mathrm{~ns}$
FIG. 7: Thermal fields at times 5 ns (a), 30 ns (b), $100 \mathrm{~ns}$ (c), and $110 \mathrm{~ns}$ (d) obtained from the electrothermal model for the RESET pulse.

Snapshot pictures of the phase-field simulations are displayed in figure 8. In the initial part of the RESET operation, the active region of the PCM material melts. As was the case for the nucleation of the solids in the isothermal simulation, the liquid phase does not appear spontaneously. Rather, the melting is initialized by introducing a liquid area around the hottest point in the structure, which is located at the interface between the PCM layer and the heater electrode (figure 8(a)). The liquid is introduced by just changing the phase fields, without modification of the concentration field. After 32 ns (b), the liquid area is growing: the GST phase melts first, followed by the grains of Ge phase. At $100 \mathrm{~ns}$, when the current stops, the size of the molten area is maximal. When the temperature starts to fall, recrystallization occurs. However, the drop in temperature leads to a drop in the interface mobility, and despite the increasing driving force, the interfaces soon get arrested (110 ns, (d)), and a large amorphous area persists in the center of the active region.

\section{SET operation}

The characteristic current for the SET operation, which leads to crystallization of the memory cell, is represented in blue in figure 6 . Snapshot pictures of the phase-field simulations are displayed in figure 9 . The initial state (a) is the same as for the RESET operation:

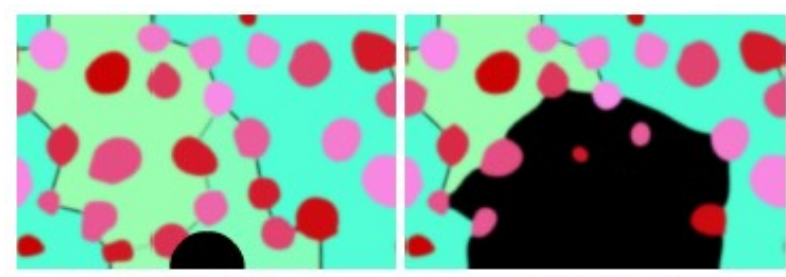

(a) $\mathrm{t}=0 \mathrm{~s}$

(b) $\mathrm{t}=32 \mathrm{~ns}$

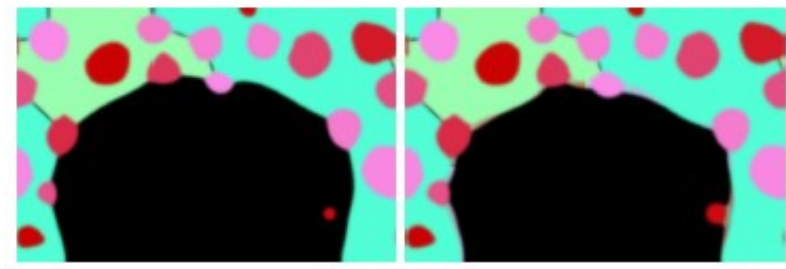

(c) $\mathrm{t}=100 \mathrm{~ns}$

(d) $\mathrm{t}=110 \mathrm{~ns}$ (final state)

FIG. 8: Snapshot pictures of the crystalline microstructure at times $0 \mathrm{~s}$ (a), $32 \mathrm{~ns}$ (b), $100 \mathrm{~ns}$ (c), and $110 \mathrm{~ns}(\mathrm{~d})$ during the RESET operation. The color code is the same as in Fig. 5.

it is obtained by introducing a liquid area at the contact with the heater. At $\mathrm{t}=100 \mathrm{~ns}$, the current starts to decrease, and consequently the temperature starts to decrease in the cell. At this time, the size of the liquid area is maximal. After $320 \mathrm{~ns}$ (b), the size of the liquid area is still large, but the regrowth of Ge and GST phases is ongoing. For quite some time, the Ge and GST phases grow together, approximately at the same speed. After $1.6 \mu \mathrm{s}$ (d), a new regime can be observed : only the GST phase grows and engulfs the Ge phase. In this stage, the GST phase releases Ge into the molten area. This enrichment in Ge causes new Ge grain to appear spontaneously at grain boundary grooves around $2.2 \mu \mathrm{s}(\mathrm{e})$. After $2.8 \mu \mathrm{s}$ (f), the recrystallization is complete.

Drastic changes in the microstructure can be noticed: after the SET operation, larger grains of Ge have been formed along the border of the area that has been molten; an area where only GST phase is present is also observed, and long and narrow grains have been formed close to the heater electrode.

These pictures indicate that the Ge distribution in the cell has been modified. In order to quantify this modification, the average germanium concentration as a function of distance from the heater electrode has been calculated by integrating the concentration field over annuli centered on the middle of the heater electrode. In order to reduce the statistical error linked to the small system size (limited number of grains), the result of seven simulations with identical parameters but different seeds for the random number generator were averaged. Figure 10 shows the difference between the initial and the final state. A clear impoverishment in Ge of the active zone can be seen, especially in the distance range from 30 to $55 \mathrm{~nm}$ from the center of the active zone. 


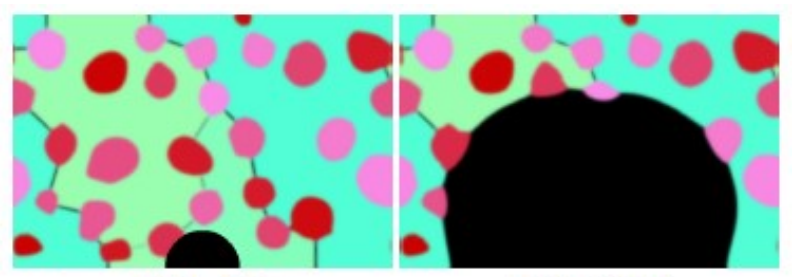

(a) $\mathrm{t}=5 \mathrm{~ns}$

(b) $\mathrm{t}=320 \mathrm{~ns}$

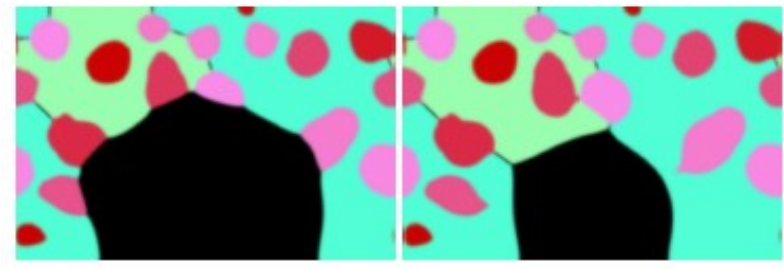

(c) $\mathrm{t}=930 \mathrm{~ns}$

(d) $\mathrm{t}=1.6 \mu \mathrm{s}$

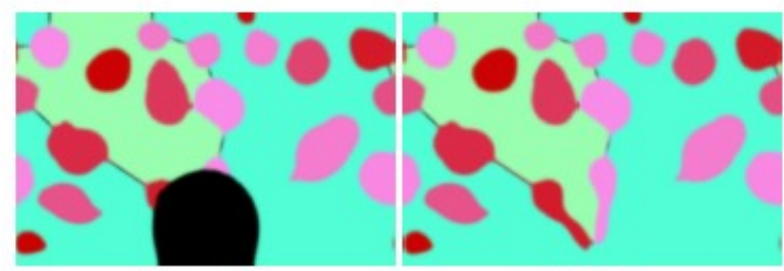

(e) $\mathrm{t}=2.2 \mu \mathrm{s}$

(f) $\mathrm{t}=2.8 \mu \mathrm{s}$

FIG. 9: Snapshot pictures of the crystalline microstructure at times $5 \mathrm{~ns}$ (a), $320 \mathrm{~ns}$ (b), $930 \mathrm{~ns}$ (c), $1.6 \mu \mathrm{s}(\mathrm{d}), 2.2 \mu \mathrm{s}(\mathrm{e})$, and $2.8 \mu \mathrm{s}$ (f) during the SET operation. The color code is the same as in figure 5 .

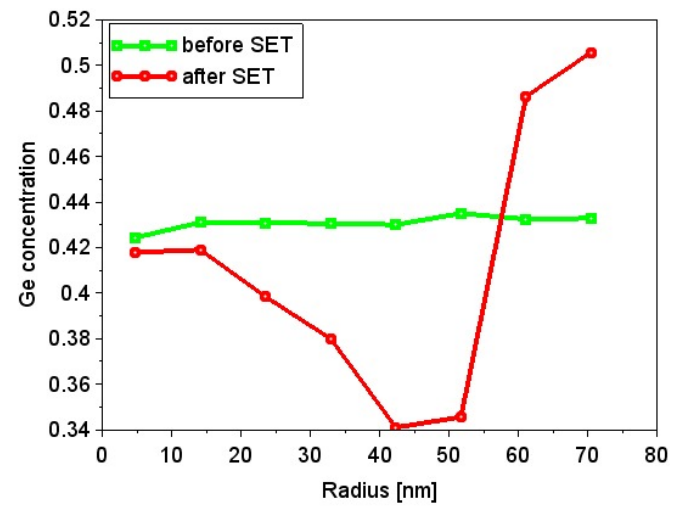

FIG. 10: Average Ge concentration after the SET operations as a function of the distance from the center of the active zone.

\section{Interface states}

In order to get a more comprehensive view of the interface states upon recrystallization during the RESET and SET operations, we have extracted the interface temperature as a function of time on the intersection of the crystal-amorphous interfaces with a vertical line located

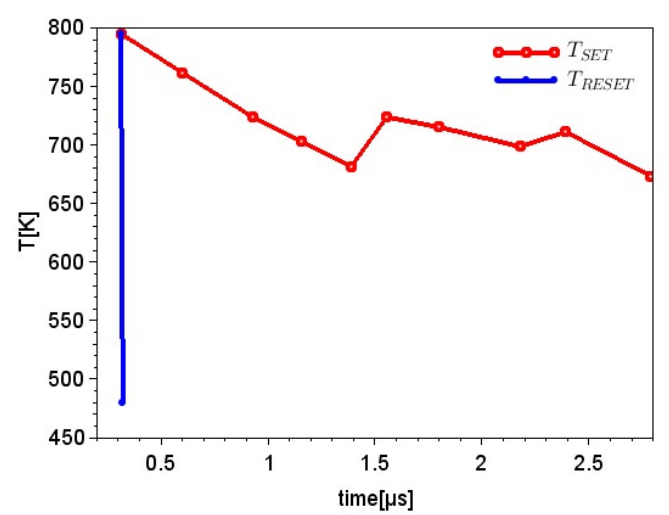

FIG. 11: Temperature at the intersection between a a vertical line separating the cell in two equal parts and the interface between crystal and liquid phases.

in the middle of the heater electrode, that is, in the center of the simulation domain. The results are displayed in Fig. 11. The blue curve, associated with the RESET operation, is represented only for the first ten nanoseconds after the stop of the electric currrent. After this short time, the temperature in the cell keeps decreasing, but the crystal-liquid interface no longer moves.

The red curve associated to the SET operation shows a decrease from $800 \mathrm{~K}$ to $680 \mathrm{~K}$, followed by a sudden rise. This is due to the fact that there is a change in the phase that occupies the fixed lateral position of our scan line on the growing interface: in the first part of the curve, the recorded temperature is the one of the Ge-amorphous interface, whereas later on it is the one of the GST phase.

These data can be plotted in a different way, which is analogous to the time-temperature-transformation (TTT) diagrams used in materials science to determine whether a certain phase will appear. In the standard TTT diagram, the temperature-time curve is compared to the characteristic waiting time for nucleation to occur. Here, we have to compare the characteristic time for recrystallization to the cooling time. A limiting curve can be obtained using the data for GST 225: in Fig. 12, we plot for each temperature the time it would take an interface in the stoichiometric compound to advance by 1 $\mu \mathrm{m}$. One can observe that, indeed, the curve for the RESET operation never enters the region delimited by this estimate. On the other hand, the curve for the SET pulse ends up inside that region.

\section{CONCLUSION AND PERSPECTIVES}

In conclusion, we have developed a coupled model for crystallization and segregation during the operations of phase-change memories that use GST enriched in germanium. This model takes into account the thermodynamics of GST, the available data on interface kinetics, and 


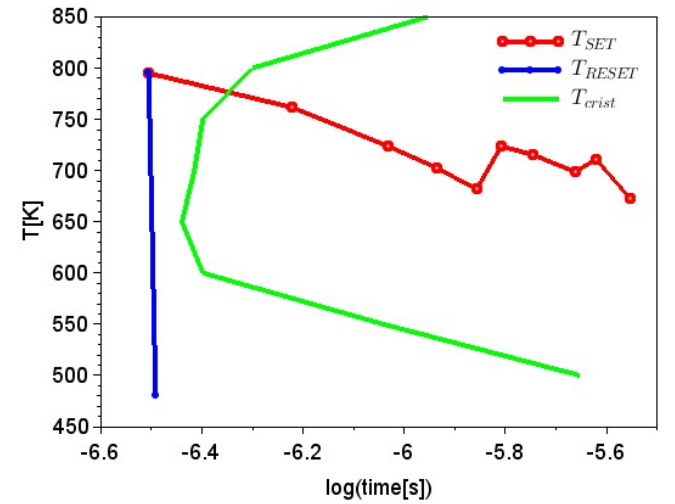

FIG. 12: Analog of a time-temperature-transformation diagram using GST225 data. The green curve indicates the time required for a GST225 crystal-liquid interface

to propagate over $1 \mu \mathrm{m}$ at a given temperature.

the polycrystalline structure of the material. The model qualitatively describes the spontaneous emergence of a two-phase polycrystalline material during the annealing of an as-deposited amorphous film. When simulations are performed with temperature fields that are typical for the conditions of device operation, the model reproduces the redistribution of alloy components that is observed in post mortem studies of memory points.

The model put forward here is in many respects only qualitative. In addition to the approximations outlined in the beginning of section 2, there are many more that have been mentioned during the exposition of the model. In addition, the input data that have been used to calibrate the model are of limited precision (e.g. the surface energy, which is a very important parameter).

Despite these caveats, our model gives a reasonable description of the processes that occur during the operation of phase-change memories with innovative materials. Even with its current limitations, the model can be useful to carry out parametric studies, which could help to better understand the role of the various physical effects that come into play. This analytic view could also serve in the future as a guide for new and more quantitative experimental analyses.

In order to transform this approach into a more quantitative tool for the development of devices, various new alleys of research could be explored. First, the pseudobinary appromixation could be relaxed; then, two concentration equations would need to be handled. Whereas this is not a problem in principle, much more detailed thermodynamic (phase diagram) and kinetic (diffusion coefficients) data would be needed to make simulations reliable. Second, the energetics of interfaces and grain boundaries could be made more realistic. In particular, the grain structure of the polycrystalline material indicates that strong interfacial anisotropy might be present. While such anisotropies can be easily modeled in the phase-field approach, the problem is again the lack of data. Such data could be obtained either from more fundamentally oriented experiments, or eventually from atomistic simulations, as has already been accomplished in metallurgy ${ }^{48}$. Third, mechanical constraints could also be taken into account, for the prize of adding a new equation (the equation for elastic displacements). Fourth, the coupling of the phase-field model and the electrothermal solver could also be improved by making it two-way: the microstructure of the material modifies the electric currents through the phase-change material and therefore also the temperature distribution. In order to include such heterogeneities, the equations for electric and heat conduction need to be resolved with the same spatial resolution as the phase-field model, with conductivities that depend on the phase fields ${ }^{49}$. Such a description on the microstructural scale would also permit to take into account the release of latent heat during interface motion in the calculation of the temperature field. Finally, it would also be interesting to simulate the long-term evolution of memory point microstructres during repeated writing cycles. This would, however, require considerable computational resources since many successive runs of the model would be nessessary.

\section{ACKNOWLEDGMENTS}

This work was financially supported by the Association Nationale Recherche Technologie (ANRT), France, and STMicroelectronics through the CIFRE contract number 2016/1237.

\section{DATA AVAILABILITY}

The data that support the findings of this study, and in particular the simulation code, are available from the corresponding author upon reasonable request.

\footnotetext{
${ }^{1}$ A. Chen, "A review of emerging non-volatile memory (NVM) technologies and applications," SOLID-STATE ELECTRONICS 125, 25-38 (2016).

${ }^{2}$ H.-S. P. Wong, S. Raoux, S. Kim, J. Liang, J. Reifenberg, B. Rajendran, M. Asheghi, and K. Goodson, "Phase change memory," Proceedings of the IEEE 98, 2201-2227 (2010), cited By 978.

${ }^{3} \mathrm{M}$. Wuttig and N. Yamada, "Phase-change materials for rewriteable data storage," Nature Materials 6, 824-832 (2007).

${ }^{4}$ S. R. Ovshinsky, "Reversible Electrical Switching Phenomena in Disordered Structures," Physical Review Letters 21, 1450-1453 (1968).

${ }^{5}$ N. Yamada, E. Ohno, N. Akahira, K. Nishiuchi, K. Nagata, and M. Takao, "High Speed Overwritable Phase Change Optical Disk Material," Japanese Journal of Applied Physics 26, 61 (1987).

${ }^{6}$ N. Yamada, E. Ohno, K. Nishiuchi, N. Akahira, and M. Takao, "Rapid phase transitions of GeTe-Sb2Te3 pseudobinary amorphous thin films for an optical disk memory," Journal of Applied Physics 69, 2849-2856 (1991).

${ }^{7}$ G. Servalli, "A $45 \mathrm{~nm}$ generation phase change memory technology," (2009) pp. 5.7.1-5.7.4.

${ }^{8}$ I. Friedrich, V. Weidenhof, W. Njoroge, P. Franz, and M. Wuttig, "Structural transformations of [formula omitted] films studied by
} 
electrical resistance measurements," Journal of Applied Physics 87, 4130-4134 (2000).

${ }^{9}$ F. Arnaud, P. Zuliani, J. Reynard, A. Gandolfo, F. Disegni, P. Mattavelli, E. Gomiero, G. Samanni, C. Jahan, R. Berthelon, O. Weber, E. Richard, V. Barral, A. Villaret, S. Kohler, J. Grenier, R. Ranica, C. Gallon, A. Souhaite, D. Ristoiu, L. Favennec, V. Caubet, S. Delmedico, N. Cherault, R. Beneyton, S. Chouteau, P. Sassoulas, A. Vernhet, Y. Le Friec, F. Domengie, L. Scotti, D. Pacelli, J. Ogier, F. Boucard, S. Lagrasta, D. Benoit, L. Clement, P. Boivin, P. Ferreira, R. Annunziata, and P. Cappelletti, "Truly innovative $28 \mathrm{~nm}$ fdsoi technology for automotive micro-controller applications embedding $16 \mathrm{mb}$ phase change memory," (2019) pp. 18.4.1-18.4.4.

${ }^{10}$ H. Y. Cheng, T. H. Hsu, S. Raoux, J. Y. Wu, P. Y. Du, M. Breitwisch, Y. Zhu, E. K. Lai, E. Joseph, S. Mittal, R. Cheek, A. Schrott, S. C. Lai, H. L. Lung, and C. Lam, "A High Performance Phase Change Memory with Fast Switching Speed and High Temperature Retention by Engineering the GexSbyTez Phase Change Material," in 2011 IEEE INTERNATIONAL ELECTRON DEVICES MEETING (IEDM) (IEEE; IEEE Electron Devices Soc (EDS), 2011) IEEE International Electron Devices Meeting (IEDM), Washington, DC, DEC 05-07, 2011.

${ }^{11}$ P. Zuliani, E. Varesi, E. Palumbo, M. Borghi, I. Tortorelli, D. Erbetta, G. D. Libera, N. Pessina, A. Gandolfo, C. Prelini, L. Ravazzi, and R. Annunziata, "Overcoming Temperature Limitations in Phase Change Memories With Optimized GeSbTe," IEEE Transactions on Electron Devices 60, 4020-4026 (2013).

${ }^{12}$ P. Zuliani, E. Palumbo, M. Borghi, G. Dalla Libera, and R. Annunziata, "Engineering of chalcogenide materials for embedded applications of Phase Change Memory," Solid-State Electronics 111, 27-31 (2015).

${ }^{13} \mathrm{M}$. Coué, "Electrical characterization and TEM study of the physical mechanisms implied in reliability issues of Ge-rich GST phase-change memories," (2016).

${ }^{14}$ W. J. Boettinger, J. A. Warren, C. Beckermann, and A. Karma, "Phase-field simulation of solidification," Annu. Rev. Mater. Res. 32, 163-194 (2002).

${ }^{15}$ I. Steinbach, "Phase-field models in materials science," Model. Simul. Mater. Sci. Eng. 17, 073001 (2009).

${ }^{16}$ M. Plapp, "Phase-Field Modelling of Solidification Microstructures," Journal of the Indian Institute of Science 96, 179-198 (2016).

${ }^{17}$ N. Provatas and K. Elder, Phase-field methods in materials science and engineering (Wiley-VCH, Weinheim, 2010).

${ }^{18}$ M. Plapp, "Phase-field models," in The Handbook of Crystal Growth, 2nd edition, Vol. 1B, edited by T. Nishinaga (Elsevier, Amsterdam, 2015) pp. 631-668.

${ }^{19}$ Y. Kwon, D.-H. Kang, K.-H. Lee, Y.-K. Park, and C.-H. Chung, "Analysis of intrinsic variation of data retention in phase-change memory using phase-field method," IEEE Electron Device Letters 34, 411-413 (2013).

${ }^{20}$ O. Cueto, V. Sousa, G. Navarro, and S. Blonkowski, "Coupling the Phase-Field Method with an electrothermal solver to simulate phase change mechanisms in PCRAM cells," in 2015 International Conference on Simulation of Semiconductor Processes and Devices (SISPAD) (IEEE, Washington DC, USA, 2015) pp. 301-304.

${ }^{21}$ F. Tabatabaei, G. Boussinot, R. Spatschek, E. Brener, and M. Apel, "Phase field modeling of rapid crystallization in the phase-change material aist," Journal of Applied Physics 122 (2017), 10.1063/1.4996429.

${ }^{22}$ R. Folch and M. Plapp, "Quantitative phase-field modeling of two-phase growth," Physical Review E 72, 011602 (2005)

${ }^{23}$ M. Plapp, "Unified derivation of phase-field models for alloy solidification from a grand-potential functional," Physical Review E 84, 031601 (2011).

${ }^{24}$ A. Choudhury and B. Nestler, "Grand-potential formulation for multicomponent phase transformations combined with thininterface asymptotics of the double-obstacle potential," Phys. Rev. E 85, 021602 (2012).
${ }^{25} \mathrm{H}$. Henry, J. Mellenthin, and M. Plapp, "Orientation-field model for polycrystalline solidification with a singular coupling between order and orientation," Physical Review B 86, 054117 (2012).

${ }^{26} \mathrm{~S}$. Bordas, M. T. Clavaguer-Mora, B. Legendre, and C. Hancheng, "Phase diagram of the ternary system Ge-Sb-Te: II. The subternary Ge-GeTe-Sb2Te3-Sb," Thermochimica Acta 107, $239-265$ (1986)

${ }^{27}$ G. Navarro, M. Coué, A. Kiouseloglou, P. Noé, F. Fillot, V. Delaye, A. Persico, A. Roule, M. Bernard, C. Sabbione, D. Blachier, V. Sousa, L. Perniola, S. Maitrejean, A. Cabrini, G. Torelli, P. Zuliani, R. Annunziata, E. Palumbo, M. Borghi, G. Reimbold, and B. De Salvo, "Trade-off between set and data retention performance thanks to innovative materials for phase-change memory," (2013) pp. 21.5.1-21.5.4, cited By 33.

${ }^{28} \mathrm{C}$. Thompson and F. Spaepen, "On the approximation of the free energy change on crystallization," Acta Metallurgica 27, 18551859 (1979), cited By 337.

${ }^{29}$ H. Lukas, S. G. Fries, and B. Sundman, Computational thermodynamics: the CALPHAD method (Cambridge University Press, Cambridge, 2007).

${ }^{30} \mathrm{M}$. Agati, F. Renaud, D. Benoit, and A. Claverie, "In-situ transmission electron microscopy studies of the crystallization of N-doped Ge-rich GeSbTe materials," MRS Communications 8, 1145-1152 (2018).

${ }^{31}$ M. Agati, C. Gay, D. Benoit, and A. Claverie, "Effects of surface oxidation on the crystallization characteristics of Ge-rich Ge-SbTe alloys thin films," Applied Surface Science 518, 146227 (2020).

32 J. Orava, A. L. Greer, B. Gholipour, D. W. Hewak, and C. E. Smith, "Characterization of supercooled liquid Ge2Sb2Te5 and its crystallization by ultrafast-heating calorimetry," Nature Materials 11, 279-283 (2012).

${ }^{33}$ S. G. Kim, W. T. Kim, and T. Suzuki, "Phase-field model for binary alloys," Physical Review E , 12 (1999).

${ }^{34} \mathrm{~S}$. M. Allen and J. W. Cahn, "A microscopic theory for antiphase boundary motion and its application to antiphase domain coarsening," Acta Metallurgica 27, 1085-1095 (1979).

${ }^{35}$ R. Kobayashi, J. A. Warren, and W. Craig Carter, "A continuum model of grain boundaries," Physica D: Nonlinear Phenomena 140, 141-150 (2000).

${ }^{36} \mathrm{R}$. Kobayashi and J.-A. Warren, "Modeling the formation and dynamics of polycrystals in 3D," Physica A 356, 127-132 (2005).

${ }^{37}$ T. Pusztai, G. Bortel, and L. Gránásy, "Phase field theory of polycrystalline solidification in three dimensions," Europhysics Letters (EPL) 71, 131-137 (2005).

${ }^{38}$ B. Korbuly, T. Pusztai, H. Henry, M. Plapp, M. Apel, and L. Gránásy, "Grain coarsening in two-dimensional phase-field models with an orientation field," Physical Review E 95, 053303 (2017).

${ }^{39}$ M. Plapp, "Remarks on some open problems in phase-field modelling of solidification," Philosophical Magazine 91, 25-44 (2011), arXiv: 1004.4502

${ }^{40}$ J. J. Hoyt, B. Sadigh, M. Asta, and S. M. Foiles, "Kinetic phase field parameters for the cu-ni system derived from atomistic computations," Acta Mater. 47, 3181 (1999).

${ }^{41}$ C. Reina, L. Sandoval, and J. Marian, "Mesoscale computational study of the nanocrystallization of amorphous Ge via a self-consistent atomistic phase-field model," Acta Materialia 77, 335-351 (2014).

${ }^{42}$ J. S. Langer, "An introduction to the kinetics of first-order phase transitions," in Solids far from equilibrium, Edition Aléa Saclay, edited by C. Godrèche (Cambridge University Press, Cambridge, UK, 1991) pp. 297-363.

${ }^{43}$ G. Novielli, A. Ghetti, E. Varesi, A. Mauri, and R. Sacco, "Atomic migration in phase change materials," in 2013 IEEE International Electron Devices Meeting (IEEE, Washington, DC, USA, 2013) pp. 22.3.1-22.3.4.

${ }^{44}$ L. S. Darken, "Diffusion, mobility and their interrelation through free energy in binary metallic systems," Trans. AIME 175, 184210 (1948). 
${ }^{45}$ L. Gránásy, T. Pusztai, and J. A. Warren, "Modelling polycrystalline solidification using phase field theory," Journal of Physics: Condensed Matter 16, R1205-R1235 (2004).

${ }^{46}$ L. Gránásy, T. Pusztai, G. Tegze, J. A. Warren, and J. F. Douglas, "Growth and form of spherulites," Phys. Rev. E 72, 011605 (2005).

${ }^{47}$ P. Zalden, K. Siegert, S. Rols, H. Fischer, F. Schlich, T. Hu, and M. Wuttig, "Specific heat of (gete)x(sb2te3) 1-x phase-change materials: The impact of disorder and anharmonicity," Chem- istry of Materials 26, 2307-2312 (2014), cited By 25.

${ }^{48}$ J. J. Hoyt, M. Asta, and A. Karma, "Atomistic and continuum modeling of dendritic solidification," Mat. Sience Eng. R 41, 121 (2003).

${ }^{49}$ A. Monas, R. Spatschek, C. Hüter, F. Tabatabaei, E. A. Brener, and M. Apel, "Phase field modeling of phase transitions stimulated by Joule heating," Journal of Crystal Growth 375, 39-48 (2013). 